\title{
A PROSPECTIVE COMPARISON STUDY BETWEENBEDSIDE ULTRASONOGRAPHY AND COMPUTED TOMOGRAPHY OF THE CHEST FOR THE DIAGNOSIS
}

OF PNEUMONIA IN EMERGENCY DEPARTMENT

Amr Abdallah El-Sayed El-Morsy, * Hassan Abd El Salam Fathy, ** Mina Montasser Gerguis, Rana Moursi Moustafa Amer

Department of Critical Care, * Department of Diagnostic and Interventional Radiology, ** Department of Emergency Medicine, Facu lty of Medicine, University of Alexandria.

\section{Introduction}

Pneumonia is considered a major healthcare problem that has a huge effect on the morbidity and mortality globally. It is considered one of the leading causes of death worldwide. Any delay or failure in the diagnosis or management of pneumonia causes significant increase in mortality. Meanwhile, inappropriate antibiotic leads to the development of antibiotic resistance. Traditionally, the diagnosis of pneumonia is

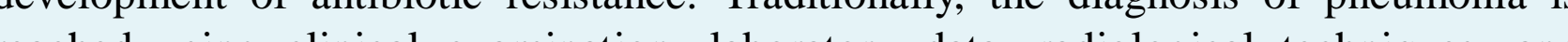
reached cultures. Clinical information alone is not enough to predict pneunonia. Chest X-ray (CXR) is the easiest approach in patients with suspected pneumonia, in search for a new infiltrate. However, studies clarified that it has low sensitivity for the diagnosis of pneumonia in comparison to chest computed tomography (CT). But the use of chest $\mathrm{CT}$ is not commonly used except for complex cases and cases with failure response to treatment. Lung ultrasonography (LUS), being a fast bedside technique, it has attracted many interests to preform studies to assess its ability to detect pleuropulmonary pathologies generally and pneumonia specifically.

\section{Aim of the work}

The aim of our study is to evaluate the sensitivity and specificity of lung ultrasound in diagnosing pneumonia in the emergency department in comparison to the CTChest as a gold standard diagnostic tool.

\section{Patients and Methods}

\section{PATIENTS}

This was a prospective observational study included 150 adult patients with clinical suspicion of pneumonia at the time of assessment with fever and at least one

unexplained respiratory complaint; dyspnea, pleuritic chest pain, cough or

hemoptysis; directly after admission to Emergency Department at Alexandria Main

University Hospital (AMUH)

\section{METHODS}

All Patients were Subjected:

1- Data Collection and Physical Examination

2- ABG analysis \& Laboratory investigations
3- Ultrasonography (US)

- Using the BLUE protocol algorithm, the emergency physician should be able to identify the profiles in which pneumonia is suspected: A-profile plus

PLAPS, A/B profile, B'-profile, and C-profile.

- In order to identify the profiles, the following signs should be recognized: Lung Sliding, A-lines, B-lines, Hepatization sign, Dynamic air bronchogram, Shred sign, and Pleural effusion.

4-Computed Tomography: Chest computed tomography (CT) was done as soon as possible (maximum 6 hours)

\section{Results}

The mean age of the patients was 52.5 years with female predominance $(56.6 \%)$. After US assessment, chest CT was done to confirm the diagnosis of pneumonia. Accordingly, they were divided into 2 groups. 103 patients $(68.67 \%)$ were diagnosed with pneumonia "Positive CT group" and 47 patients (31.33\%) were not diagnosed with pneumonia "Negative CT group". 91 patients in the positive CT groups had one of the four main profiles: A + PLAPS profile was present in 39 patients $(37.9 \%)$, followed by A/B profile with 21 patients $(21.4 \%)$, then $\mathrm{C}$ profile with $22(20.4 \%)$ and last was B' profile with $9(8.7 \%)$.

Other profiles were present in 12 patients of the same group: B profile was present in 7 patients $(6.8 \%), \mathrm{A}+$ no PLAPS was in 5 patients $(4.9 \%)$ and $\mathrm{A}^{\prime}$ profile was absent in all patients with pneumonia.

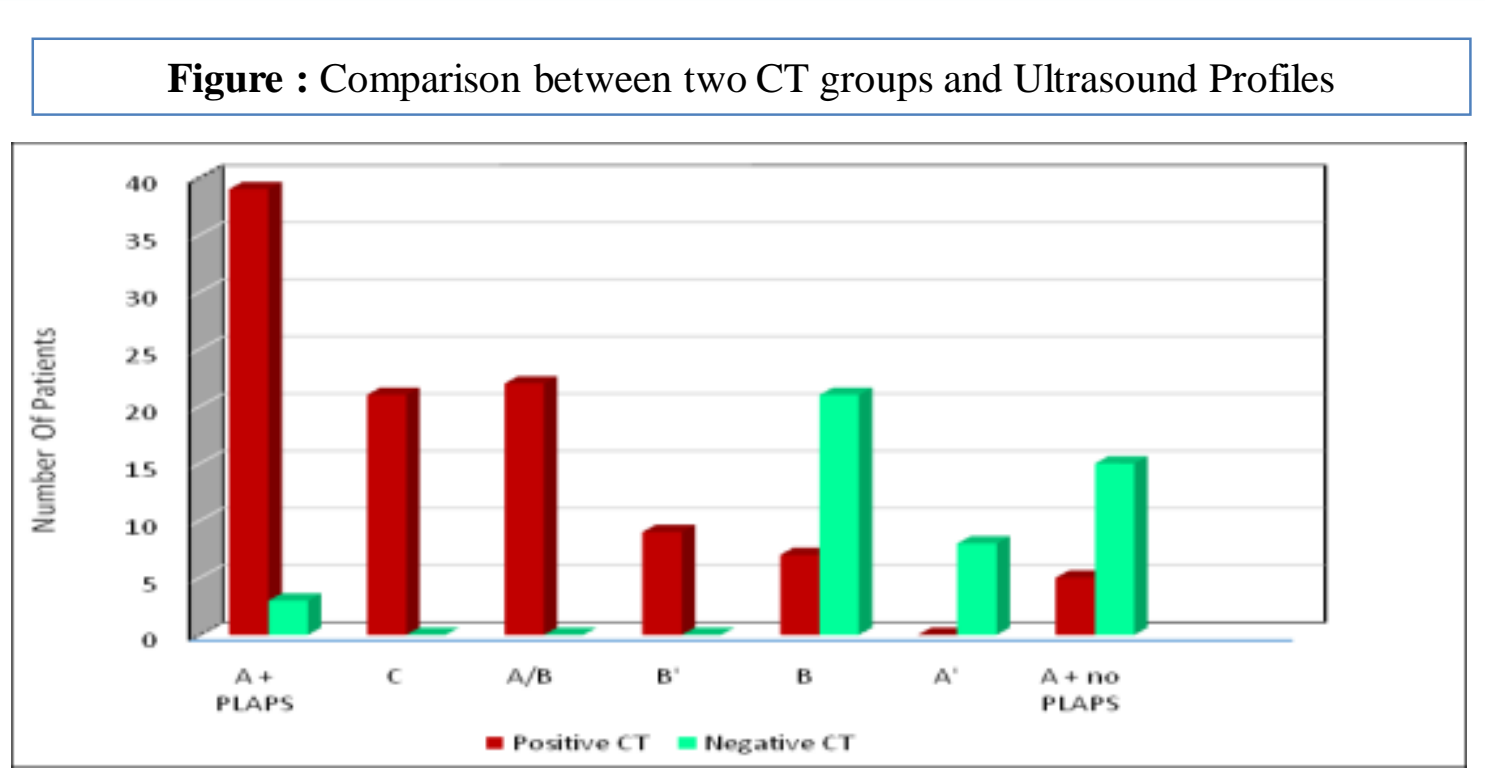

Table: Sensitivity and specificity for U/S and profiles suggestive of pneumonia according to CT pneumonia

\begin{tabular}{|l|c|c|}
\hline \multicolumn{1}{|c|}{ US } & Sensitivity & Specificity \\
\hline A+ PLAPS & 37.86 & 93.62 \\
\hline A/B & 21.36 & 100.0 \\
\hline C & 20.39 & 100.0 \\
\hline B & 8.74 & 100.0 \\
\hline U/S suggestive of pneumonia & 88.35 & 93.62 \\
\hline
\end{tabular}

\section{Conclusion}

According to the results of this study, emergency ultrasonography showed high sensitivity and specificity, so it is considered a bedside, reliable, rapid and non-invasive technique. The results suggest that it could have a significant role in the diagnostic workup of pneumonia in the ED, alongside with the computed tomography, in emergency settings. 